\title{
Neutrons aged in the bottle
}

Knowing the half-life of the neutron is important for particle physicists and cosmologists alike. A new technique should soon improve the accuracy of measurements to better than the present half per cent.

THE value of the fine-structure constant of electromagnetic theory is known to an accuracy of about one part in ten million. In recent measurements of the magnetic moment of the electron, whose small departure from the classical value constitutes a powerful diagnostic test of quantum electrodynamics, the error comes only in the tenth decimal place. So it may come as something of a surprise to discover that the best current estimate of the lifetime of the neutron, which was the third 'elementary' particle to be discovered, is 891.6 seconds with an error of \pm 5.1 seconds an accuracy of no better than half a per cent.

The error should not be taken as indicative of any lack of effort on the part of experimenters. Measurements of the neutron's half-life are constantly being attempted by groups all around the world, and the magnitude of the 5-second error represents more than anything else a measure of the difficulty that these different groups have in coming up with consistent values. On the face of it, a new value given in the 7 August issue of Physical Review Letters $(63,593 ; 1989)$ by W. Mampe et al., five physicists from France, the United Kingdom and the United States, does not improve things markedly: their new estimate is $887.6 \pm 3$ seconds.

This is certainly consistent with the previous best guess, but the error is much the same. What is of interest in the new measurement is the manner in which it was obtained, using stored ultracold neutrons, and the promise it holds for future determinations.

The neutron decays, when not bound up in a stable nucleus, into a proton, an electron and a neutrino. As such, it can be considered the fundamental manifestation of radioactive beta-decay, and therein lies its significance for elementary particle physics. Enrico Fermi, before he moved to the United States and created the first self-sustaining nuclear reaction, was responsible for a theoretical analysis of beta decay that survives today in standard accounts of weak interaction theory.

Fermi introduced a new strength parameter, which measured the magnitude of the then-unknown interaction responsible for beta decay. This kind of nuclear instability was later interpreted as evidence of a new force of nature, the weak interaction, which has itself now been subsumed into the unified electroweak interaction of Glashow, Salam and Weinberg.
Nevertheless, Fermi's constant, or a slightly different formulation of it, has remained as the fundamental constant of the weak interaction, as Newton's constant remains the basic parameter of Einstein's theory of gravity. And measurement of the neutron's half-life remains one of the few comparatively simple ways for experimenters to get at the value of this number. (Radioactive half-lives of large nuclei can be measured with good accuracy by obtaining a lump of the appropriate substance and counting decay products, but the relation of the weak interaction strength to measured life-time is obscured by the complication of manybody nuclear physics.)

On top of its intrinsic importance to fundamental physics, the value of the halflife of the neutron also has an anthropic significance in cosmology. At about one second old, the Universe consists of protons, neutrons, electrons and radiation, with protons and neutrons having roughly equal numbers.

The radiation temperature is then too high to allow protons and neutrons to get together as deuterium nuclei and thence form heavier elements, but after three minutes, deuterium can survive and nucleosynthesis can proceed.

To a first approximation, all the neutrons that are around at a cosmic age of three minutes end up in ${ }^{4} \mathrm{He}$ nuclei. If the neutron were stable, and at this critical age were still as abundant as protons, then every two neutrons would team up with a pair of protons to form a ${ }^{4} \mathrm{He}$ nucleus, and nothing would be left over. But the neutron's instability reduces its numbers enough, by the time 180 seconds have been ticked off, that only about a quarter of the Universe's raw material is cooked into ${ }^{4} \mathrm{He}$, leaving enough hydrogen around, along with a few other trace elements, for stars and galaxies to begin forming. As a nuclear fuel, hydrogen is considerably more inflammable than helium, and stellar evolution is able ultimately to manufacture the heavy elements of which planets and their inhabitants are made.

The imprecision with which the neutron's half-life is known is thus no reflection of the urgency of the need to know. The difficulties are practical ones. A standard technique in many earlier experiments was to measure the rate at which a beam of neutrons emitted electrons. Counting electrons is easy: charged particles can be siphoned off with suitable magnetic fields, and detectors are efficient. But to determine a decay constant from such an experiment, the number of neutrons needs also to be known, and this is the source of inaccuracy. Neutron detectors are not very efficient, and because the motion of neutrons cannot be controlled by electromagnetic fields, particles can drift out of the beam without the experimenter being able to tell.

In the technique used by $\mathrm{W}$. Mampe and P. Ageron of the Institut LaueLangevin in Grenoble, France, and their colleagues, a source of ultracold neutrons (with kinetic energies less than one micro electron volt) is used to fill a neutron 'bottle'. To measure the half-life, the number of neutrons has to be measured going in, and at some later time, and as the same detector is used for both measurements the lack of detector efficiency becomes irrelevant.

But this method, which has become possible only with the advent of ultracold neutron sources during the past couple of years, still requires a good deal of delicacy. The neutrons are confined to a small volume, some tens of centimetres on a side, made of glass plates coated with a viscous heavy oil which both seals in the vacuum and reflects neutrons with high efficiency.

The rate at which neutrons are lost is a combination of decays and absorption by the walls, and the latter must be estimated and subtracted to yield a true half-life. Moreover, during the several thousand seconds through which the neutrons are confined, collisions with the oil-coated walls gradually reduce the average energy of the neutrons, and gravity causes them to settle slowly to the bottom. As the experiment proceeds, the physical distribution of the neutrons in the glass box changes, which introduces a systematic error in neutron counts taken at different times. This indeed proves to be the biggest source of error in the measurement.

But because this problem is fairly simple to understand, it is susceptible to revised experimental design and more sophisticated calculations of the loss rate. Undoubtedly other errors will intrude as the experiment is improved, but what Mampe et al. have demonstrated is that a straightforward realization of this technique is at least as good as the most refined implementations of earlier methods.

David Lindley 